\title{
Secagem convencional de casca de mandioca proveniente de resíduos de indústria de amido ${ }^{1}$
}

\author{
Divina Aparecida Anunciação Vilhalva ${ }^{2}$, Manoel Soares Soares Júnior ${ }^{2}$, Márcio Caliari² $^{2}$, Flávio Alves da Silva²
}

\begin{abstract}
Conventional drying of cassava peel from starch industry waste

Cassava peels from starch industry waste are highly perishable. The drying process is an alternative way to provide a safe storage, free from the development of microorganisms, for the production of flour destined to human consumption. This study aimed at evaluating the kinetics and modeling of the cassava peels drying process, with the aid of a central composite rotational experimental design. Eleven tests were performed with different temperatures $\left(53^{\circ} \mathrm{C}, 55^{\circ} \mathrm{C}, 60^{\circ} \mathrm{C}, 65^{\circ} \mathrm{C}\right.$ and $\left.67^{\circ} \mathrm{C}\right)$ and air flow rates $\left(0.0159 \mathrm{~m}^{3} \mathrm{~kg}^{-1} \mathrm{~s}^{-1}, 0.0166 \mathrm{~m}^{3} \mathrm{~kg}^{-1} \mathrm{~s}^{-1}\right.$, $0.0183 \mathrm{~m}^{3} \mathrm{~kg}^{-1} \mathrm{~s}^{-1}, 0.0199 \mathrm{~m}^{3} \mathrm{~kg}^{-1} \mathrm{~s}^{-1}$ and $\left.0.0206 \mathrm{~m}^{3} \mathrm{~kg}^{-1} \mathrm{~s}^{-1}\right)$. The drying process was carried out in a conventional dryer tray and the drying times, instrumental color parameters (lightness - L*; $\mathrm{a}^{*}$ and $\mathrm{b}^{*}$ coordinates), titratable acidity and $\mathrm{pH}$ of the flour samples obtained after grinding the dehydrated cassava peels from each drying test were evaluated. The temperature increase resulted in a tendency to bleach the material, and a slight variation was observed for titratable acidity and $\mathrm{pH}$ in the samples. Temperature and air flow speed affected the drying kinetics, since their increase reduced the time for drying the cassava peels. The minimum drying time (420 min.) was reached when the dryer temperature was set to $67^{\circ} \mathrm{C}$ and the air flow to $0.0183 \mathrm{~m}^{3} \mathrm{~kg}^{-1} \mathrm{~s}^{-1}$. This was considered the ideal setting, because it minimized the processing time, without altering the product features. The Crank model for plane geometry fitted well the experimental data obtained from the cassava peel drying process.
\end{abstract}

KEY-WORDS: Manihot esculenta Crantz; drying kinetics; cassava peel flour; agro-industrial waste.

\section{INTRODUÇÃO}

A cultura da mandioca (Manihot esculenta Crantz) é amplamente disseminada no Brasil, não apresentando restrições, quanto às condições de solo e clima, podendo ser cultivada em todas as regiões do País (Cereda 2001, Souza \& Fialho 2003). A planta é um arbusto perene e apresenta sistema radicular

\section{RESUMO}

Cascas de mandioca provenientes de resíduos de fecularias são altamente perecíveis. A secagem apresentase como alternativa na armazenagem segura e livre do desenvolvimento de micro-organismos, para a produção de farinha com aplicabilidade na alimentação humana. Este trabalho objetivou estudar a cinética e modelagem da secagem de cascas de mandioca, utilizando-se planejamento experimental central composto rotacional. Foram realizados 11 testes, empregando-se diferentes temperaturas $\left(53^{\circ} \mathrm{C}, 55^{\circ} \mathrm{C}, 60^{\circ} \mathrm{C}, 65^{\circ} \mathrm{C}\right.$ e $\left.67^{\circ} \mathrm{C}\right)$ e fluxos de $\operatorname{ar}\left(0,0159 \mathrm{~m}^{3} \mathrm{~kg}^{-1} \mathrm{~s}^{-1} ; 0,0166 \mathrm{~m}^{3} \mathrm{~kg}^{-1} \mathrm{~s}^{-1} ; 0,0183 \mathrm{~m}^{3} \mathrm{~kg}^{-1} \mathrm{~s}^{-1}\right.$; $0,0199 \mathrm{~m}^{3} \mathrm{~kg}^{-1} \mathrm{~s}^{-1}$; e $0,0206 \mathrm{~m}^{3} \mathrm{~kg}^{-1} \mathrm{~s}^{-1}$ ). A secagem foi realizada em secador convencional de bandejas, sendo determinados os tempos de secagem, parâmetros instrumentais de cor (luminosidade $\mathrm{L}^{*}$; coordenadas $\mathrm{a}^{*} \mathrm{e} \mathrm{b}^{*}$ ), acidez titulável e $\mathrm{pH}$ das amostras de farinha obtidas após moagem das cascas desidratadas de cada ensaio de secagem. Observou-se tendência ao clareamento do material, com o aumento da temperatura. Ocorreu pequena variação nos valores de acidez titulável e pH das amostras analisadas. A temperatura e a velocidade do ar influenciaram na cinética de secagem, pois, com o aumento de ambas, o tempo de secagem das cascas foi reduzido. O tempo mínimo de secagem (420 min.) foi obtido quando a temperatura do secador foi ajustada para $67^{\circ} \mathrm{C}$ e o fluxo de ar para $0,0183 \mathrm{~m}^{3} \mathrm{~kg}^{-1} \mathrm{~s}^{-1}$. Este foi considerado o ajuste ideal, pois minimizou o tempo de processamento, sem alterar as características do produto. O modelo de Crank para geometria plana ajustou-se bem aos dados experimentais obtidos na secagem da casca de mandioca.

PALAVRAS-CHAVE: Manihot esculenta Crantz; cinética de secagem; farinha de casca de mandioca; resíduo agroindustrial.

subterrâneo espessado (raízes tuberosas) bastante resistente à seca, o qual acumula grande quantidade de amido (El-Sharkawy 2006).

Além do alto consumo in natura (cozida ou frita), ou na forma de farinhas, a mandioca apresenta grande importância para a indústria farmacêutica, química, têxtil e de papel (Vilpoux \& Cereda 1995, Marques et al. 2000, Dias \& Leonel 2006).

1. Trabalho recebido em jun./2012 e aceito para publicação em set./2012 (n registro: PAT 19065).

2. Universidade Federal de Goiás (UFG), Escola de Agronomia e Engenharia de Alimentos, Setor de Engenharia de Alimentos, Goiânia, Goiás, Brasil.E-mails: divinavilhalva@yahoo.com.br, mssoaresjr@hotmail.com, macaliari@ig.com.br, flaviocamp@gmail.com. 
A casca de mandioca é um dos resíduos gerados durante o processamento da fabricação da farinha ou fécula, sendo constituída, além das cascas propriamente ditas (película marrom), por entrecascas, detritos do córtex e pontas de raízes, e apresentando alto teor de umidade (85\%) (Cereda 1996).

De acordo com Prado et al. (2000), a casca de mandioca desidratada apresenta $58,1 \%$ de amido, $3,4 \%$ de proteína bruta e $28,6 \%$ de fibra, em detergente neutro. Estes resíduos são ricos em amido e apresentam fibras de boa qualidade (Cereda 1996, Lacerda et al. 2009), podendo ser transformados em produtos alimentícios (Souza 2011, Vilhalva et al. 2011). No entanto, o que limita a utilização destes é o seu alto teor de umidade. Sendo assim, a secagem representa uma alternativa para a preservação e aproveitamento alimentício da casca de mandioca, resíduo da indústria processadora de mandioca.

Segundo Corrêa et al. (2008), a secagem é empregada visando, principalmente, à conservação de produtos perecíveis, pois permite diminuir o teor de água e o armazenamento por período mais longo. A secagem também impede o crescimento de micro-organismos e insetos e reduz a massa e o volume a ser transportado (Andrade et al. 2006).

Dentre os vários processos de secagem de produtos alimentícios, a cinética e a modelagem matemática são ferramentas importantes e muito utilizadas na otimização destes processos (Afonso Júnior \& Corrêa 1999, Martinazzo et al. 2007, Ferreira \& Pena 2010, Resende et al. 2010).

A cinética de secagem é um assunto vastamente coberto pela literatura, para diferentes produtos. Evidencia-se que, dentre as variáveis mais importantes, estão a temperatura e o fluxo e/ou velocidade do ar utilizados (Queiroz \& Nebra 2001, Demirel \& Turhan 2003, Krokida et al. 2003, Lahsasni et al. 2004, Giraldo-Zuniga et al. 2006, Corrêa et al. 2007, Kaya et al. 2008, Nagle et al. 2008).

A modelagem da secagem é fundamental para a seleção e otimização das condições de processo. As taxas de secagem devem ser relacionadas para um determinado produto e para uma determinada operação (processo e equipamento), podendo ser estabelecidas mediante estudos de transferência de calor e massa, além dos possíveis mecanismos de migração interna de umidade. Entretanto, as equações de transferência de calor e massa demandam considerável conhecimento de cálculo numérico, para sua solução analítica (Simal et al. 1997, Park et al. 2002, Doymaz 2004, Simal et al. 2005).

Este trabalho objetivou estudar a cinética da secagem da casca de mandioca, por meio do controle das variáveis de processo (temperatura e fluxo do ar), a fim de garantir o menor tempo de processo e a qualidade física (cor) e físico-química (pH e acidez), visando à utilização das cascas de mandioca provenientes de descartes de fecularia na alimentação humana.

\section{MATERIAL E MÉTODOS}

O experimento foi realizado na Escola de Agronomia e Engenharia de Alimentos da Universidade Federal de Goiás, em Goiânia (GO), em 2011, com cascas de mandioca (CM) da cultivar Iapar-12, doadas pela Fecularia de Bela Vista Ltda. (Febela), situada no município de Bela Vista de Goiás (GO). $\mathrm{Na}$ indústria, as $\mathrm{CM}$ foram coletadas diretamente na saída da área de lavagem e descascamento, utilizando-se sacos de polietileno de baixa densidade. As CM foram imersas em solução de hipoclorito de sódio Cromoline $^{\circledR}$, na concentração de $200 \mathrm{mg} \mathrm{L}^{-1}$, por $15 \mathrm{~min}$. Os valores foram confirmados com fitas de cloro-teste Hidroall ${ }^{\circledR}$.

Utilizou-se, para o estudo de otimização da secagem das CM, um secador convectivo de bandejas (1,90 $\mathrm{m}$ de altura por $0,80 \mathrm{~m}$ de largura, com capacidade de cinco bandejas metálicas de $55 \mathrm{~cm}$ x $57 \mathrm{~cm}$ cada), com controle de temperatura e de fluxo do ar. As variáveis de secagem utilizadas para obtenção das diferentes farinhas de cascas de mandioca (FCM), nas diferentes condições experimentais, foram a temperatura do ar $\left(\mathrm{T}_{\mathrm{Ar}}\right)$, em ${ }^{\circ} \mathrm{C}$, e o fluxo de $\operatorname{ar}\left(\mathrm{F}_{\mathrm{Ar}}\right)$, em $\mathrm{m}^{3} \mathrm{~kg}^{-1} \mathrm{~s}^{-1}$. Utilizou-se delineamento central composto rotacional (Tabela 1) porque os esquemas fatoriais fracionários permitem a diminuição do número de ensaios e, por consequência, de análises.

Para o levantamento das curvas de cinética de secagem da CM, 1,0 kg de amostra úmida foi acondicionado na bandeja do equipamento de secagem e os valores de $\mathrm{T}_{\mathrm{ar}}$ e $\mathrm{F}_{\mathrm{ar}}$ (variáveis independentes) foram ajustados de acordo com o respectivo experimento (Tabela 1). A umidade inicial das amostras foi determinada segundo metodologia do Instituto Adolfo Lutz (2005). Durante o processo, a cada 30 min., as amostras de CM foram movimentadas, para que o produto se tornasse homogêneo, além de a massa ser determinada em balança semianalítica. Tendo como 
Tabela 1. Planejamento experimental utilizado para avaliar a influência da temperatura $\left(\mathrm{T}_{\mathrm{Ar}}\right)$ e fluxo do ar $\left(\mathrm{F}_{\mathrm{Ar}}\right)$ (níveis codificados e reais) no tempo de secagem da casca de mandioca e nas características de cor, $\mathrm{pH}$ e acidez das farinhas obtidas após a moagem (Goiânia, GO, 2011).

\begin{tabular}{ccccc}
\hline & \multicolumn{2}{c}{$\begin{array}{c}\text { Variáveis } \\
\text { codificadas }\end{array}$} & \multicolumn{2}{c}{ Valores reais } \\
\cline { 2 - 5 } Ensaio & $\mathrm{X}_{1}$ & $\mathrm{X}_{2}$ & $\mathrm{~T}_{\mathrm{Ar}}\left({ }^{\circ} \mathrm{C}\right)$ & $\mathrm{F}_{\mathrm{Ar}}\left(\mathrm{m}^{3} \mathrm{~kg}^{-1} \mathrm{~s}^{-1}\right)$ \\
\hline E01 & -1 & -1 & 55 & 0,0166 \\
E02 & 1 & -1 & 65 & 0,0166 \\
E03 & -1 & 1 & 55 & 0,0199 \\
E04 & 1 & 1 & 65 & 0,0199 \\
E05 & $-1,41$ & 0 & 53 & 0,0183 \\
E06 & 1,41 & 0 & 67 & 0,0183 \\
E07 & 0 & $-1,41$ & 60 & 0,0159 \\
E08 & 0 & 1,41 & 60 & 0,0206 \\
E09 & 0 & 0 & 60 & 0,0183 \\
E10 & 0 & 0 & 60 & 0,0183 \\
E11 & 0 & 0 & 60 & 0,0183 \\
\hline
\end{tabular}

referência a massa e o teor de umidade inicial obtido para cada amostra, o experimento cessou quando a umidade final da casca atingiu teor igual ou inferior a $0,1 \mathrm{~kg}(\mathrm{~kg})^{-1}$ (base úmida), já que o nível de umidade adequado para o armazenamento de farinhas deve ser inferior a $0,14 \mathrm{~kg} \mathrm{~kg}^{-1}$ (Brasil 2005), mas optou-se por um nível ainda mais seguro, por se tratar de uma aplicação para alimentação humana e a FCM ser constituída por resíduo proveniente do solo.

Após os ensaios de secagem, cada amostra de CM desidratada foi triturada em moinho (Marconi, MA090 CET, Piracicaba, Brasil) com peneira de 30 mesh, para obtenção da farinha de CM (FCM). Como variáveis dependentes (respostas), utilizaram-se o tempo de secagem da CM $(\mathrm{t})$, parâmetros instrumentais de cor (luminosidade - $\mathrm{L}^{*}$; coordenadas de cromaticidade $\mathrm{a}^{*}$ e $\left.b^{*}\right)$ e $\Delta \mathrm{E}$ da acidez titulável e do $\mathrm{pH}$ da FCM.

$\mathrm{O}$ valor do $\mathrm{pH}$ de cada amostra foi aferido utilizando-se potenciômetro digital (Hanna Instruments, HI9224). Tanto o pH, como a acidez titulável das amostras de FCM, foram determinados conforme metodologias recomendadas pelo Instituto Adolfo Lutz (2005). A leitura dos parâmetros instrumentais de cor foi realizada em colorímetro (Hunter Lab Color Quest II, Reston, Estados Unidos), utilizando-se o sistema L*, $\mathrm{a}^{*} \mathrm{e} \mathrm{b}^{*} \mathrm{CIE}$ (Commission Internacional de L' Eclairage), onde o $\mathrm{L}^{*}$ representa a luminosidade, em escala de 0 (preto) a 100 (branco); a* uma escala de tonalidades de vermelho $(0+a)$ a verde $(0$ - a); e b* uma escala de tonalidades de amarelo
$(0+$ b) a azul $(0-b)$. O valor do $\Delta \mathrm{E}$ foi calculado pela formula $\Delta \mathrm{E}=\left[\left((\Delta \mathrm{L})^{2}+(\Delta \mathrm{a})^{2}+(\Delta \mathrm{b})^{2}\right)^{0,5}\right]$, conforme descrito por Álvares et al. (2003).Todas as análises foram realizadas em triplicata.

Os dados obtidos foram avaliados por análise de variância, sendo determinados os coeficientes dos termos dos modelos de regressão e construídos os gráficos de curvas de contorno, utilizando-se o programa Statistica 7.0 (Statsoft 2007).

A teoria da migração de água por difusão, baseada na segunda lei de Fick, foi considerada no estudo da cinética de secagem. Considerando-se que a massa dos resíduos de casca de mandioca pode ser representada por uma placa plana, foi utilizada a equação proposta por Crank (1975):

$$
k=\frac{D_{e f} \pi^{2}}{L^{2}}
$$

sendo $k=$ constante de secagem, $D_{e f}=$ difusidade efetiva e $L=$ dimensão característica, a fim de se realizar a modelagem da cinética de secagem.

O modelo matemático de secagem do presente trabalho levou em conta a transferência de massa com as seguintes considerações: taxa decrescente e geometria plana, transporte unidirecional, densidade da amostra durante a secagem não dependente da posição, distribuição inicial de densidade uniforme, material homogêneo, processo aproximadamente isotérmico, distribuição da umidade inicial no material uniforme, difusividade efetiva constante e desprezíveis resistências externas ao transporte de massa.

$\mathrm{O}$ ajuste do modelo aos dados experimentais da cinética de secagem foi realizado mediante procedimento de regressão linear e não linear do pacote Statistica 7.0 (Statsoft 2007). O critério de escolha do modelo mais ajustado às curvas cinéticas incluiu a determinação do coeficiente de regressão $\left(R^{2}\right)$ entre as respostas observadas e os valores preditos pelo modelo (Barros Neto et al. 1996) e o módulo do desvio relativo médio $(P)$, cuja definição encontra-se descrita na seguinte equação (Lomauro et al. 1985):

$$
P=\frac{100}{N} \sum_{i=1}^{N} \frac{\left|V_{P}-V_{0}\right|}{V_{0}}
$$

na qual $P=$ desvio relativo médio (\%), $V_{p}=$ valores preditos pelo modelo, $V_{0}=$ valores observados experimentalmente e $N=$ número de pontos experimentais. 


\section{RESULTADOS E DISCUSSÃO}

Na Tabela 2, podem ser observados os tempos de secagem obtidos nos ensaios realizados no secador convectivo. Na Figura 1, encontram-se os ajustes das curvas de secagem da casca de mandioca (CM) para todos os ensaios do planejamento (Tabela 1), onde podem ser visualizados, por meio de curvas, os termos da umidade adimensional experimental e do modelo difusional de Cranck versus o tempo de secagem. Observou-se que a temperatura do ar $\left(\mathrm{T}_{\mathrm{ar}}\right)$ e o fluxo do ar $\left(\mathrm{F}_{\mathrm{ar}}\right)$ influenciaram na cinética de secagem $(p \leq 0,05)$, pois, com o aumento de ambas as variáveis, diminuiu o tempo de secagem da CM. A média do tempo de secagem, nas condições do ponto central, foi de $440 \mathrm{~min}$., com desvio-padrão de 34,64 min. e coeficiente de variação de 7,9\%, o que pode ser, provavelmente, explicado devido a erros experimentais não controlados, como falta de homogeneidade da CM, revolvimento manual, variação da umidade relativa do ar, etc.

$\mathrm{Na}$ Figura 2, pode ser visualizada a taxa de secagem, em função da umidade (em base seca) da CM desidratada com ar quente, para as várias combinações de $\mathrm{T}_{\mathrm{ar}}$ e $\mathrm{F}_{\mathrm{ar}}$ utilizadas neste trabalho.

Observou-se que as taxas de secagem foram mais altas no início do processo de secagem, quando o conteúdo de umidade era maior (Região II). Após os períodos iniciais do processo de secagem, ocorreu um rápido declínio nas taxas de secagem, para todos os experimentos (Região I). Em seguida, abaixo de $1,5 \mathrm{~kg}$ de água por $\mathrm{kg}$ de matéria seca (linha tracejada da Figura 2), as curvas da taxa de secagem continuaram a cair, mas de forma gradual e lenta, quase de forma linear, até as condições de equilíbrio. De fato, abaixo deste conteúdo crítico de umidade, as diferenças nas taxas de secagem entre os ensaios não foram muito claramente observadas.

Constatou-se que, quanto maior a temperatura, maior a taxa de secagem. Esta afirmação ficou evidente nos ensaios E02, E04 e E06. Resultados semelhantes foram observados por Ferreira \& Pena (2010), em estudo sobre secagem da casca do maracujá amarelo.

As modelagens das curvas de secagem foram realizadas com os dez primeiros termos da série, encontrados pelo uso do modelo. Na Tabela 3, foram apresentados os valores obtidos para difusividade efetiva $\left(D_{e f}\right)$, coeficiente de correlação $\left(R^{2}\right)$ e desvio relativo médio $(P)$, para cada experimento do planejamento. Para determinar a difusividade efetiva, utilizou-se o software Statistica 7.0 (Statsoft 2007), considerando-se que o produto final era uma placa plana e utilizando-se o método de ajuste não linear, bem como tomando-se os N primeiros termos da solução exata (equação 1) do modelo de Fick, para esta geometria. Utilizou-se espessura média $(L)$ da placa igual a $0,00945 \mathrm{~m}$, obtida pela média da medida da espessura de 100 placas, e obteve-se o melhor ajuste para $\mathrm{N}=10$, pelo critério do $R^{2}$ mais próximo de 1 .

Percebeu-se que o modelo difusional de Crank não apresentou bom ajuste aos dados experimentais, pois os valores de $P$ obtidos foram superiores (Tabela 3) ao valor sugerido por Aguerre et al. (1989), que é de $10 \%$, apesar de apresentar coeficiente de correlação próximo da unidade. O aumento da variável temperatura, nos ensaios de secagem, favoreceu o processo de transferência de massa e, consequen-

Tabela 2. Tempo de secagem $\left(\mathrm{t}_{\mathrm{s}}\right)$ e valores referentes às umidades iniciais $\left(\mathrm{X}_{0}\right)$ e finais $\left(\mathrm{X}_{\mathrm{f}}\right)$ das amostras de casca de mandioca, em base seca, em função do fluxo do ar $\left(\mathrm{F}_{\mathrm{ar}}\right)$ e da temperatura do $\operatorname{ar}\left(\mathrm{T}_{\mathrm{ar}}\right)$, além da umidade relativa do ar $\left(\mathrm{RH}_{\mathrm{ar}}\right)(\mathrm{Goiânia}, \mathrm{GO}, 2011)$.

\begin{tabular}{ccccccc}
\hline \multirow{2}{*}{ Ensaio } & $\mathrm{RH}_{\mathrm{ar}}$ & $\mathrm{T}_{\mathrm{ar}}$ & $\mathrm{F}_{\mathrm{ar}}$ & $\mathrm{X}_{0 \text { casca }}$ & $\mathrm{X}_{\mathrm{f} \text { casca }}$ & $\mathrm{t}_{\mathrm{s}}$ \\
\cline { 2 - 7 } & $\%$ & ${ }^{\circ} \mathrm{C}$ & $\mathrm{m}^{3} \mathrm{~kg}^{-1} \mathrm{~s}^{-1}$ & $\mathrm{~kg}_{\mathrm{w}} / \mathrm{kg}_{\mathrm{s}}$ & $\mathrm{kg}_{\mathrm{w}} / \mathrm{kg}_{\mathrm{s}}$ & $\mathrm{min}$ \\
\hline E01 & 58,2 & $55 \pm 0,5$ & 0,0166 & 2,45 & 0,06 & 600 \\
E02 & 55,5 & $65 \pm 0,5$ & 0,0166 & 2,85 & 0,11 & 450 \\
E03 & 62,6 & $55 \pm 0,5$ & 0,0199 & 2,33 & 0,06 & 570 \\
E04 & 64,6 & $65 \pm 0,5$ & 0,0199 & 2,85 & 0,11 & 420 \\
E05 & 59,5 & $53 \pm 0,5$ & 0,0183 & 2,33 & 0,10 & 510 \\
E06 & 67,8 & $67 \pm 0,5$ & 0,0183 & 2,85 & 0,09 & 420 \\
E07 & 57,9 & $60 \pm 0,5$ & 0,0159 & 2,85 & 0,11 & 540 \\
E08 & 65,9 & $60 \pm 0,5$ & 0,0206 & 2,85 & 0,11 & 540 \\
E09 & 63,3 & $60 \pm 0,5$ & 0,0183 & 2,85 & 0,11 & 420 \\
E10 & 50,9 & $60 \pm 0,5$ & 0,0183 & 2,33 & 0,10 & 420 \\
E11 & 64,9 & $60 \pm 0,5$ & 0,0183 & 2,85 & & 0,11 \\
\hline
\end{tabular}



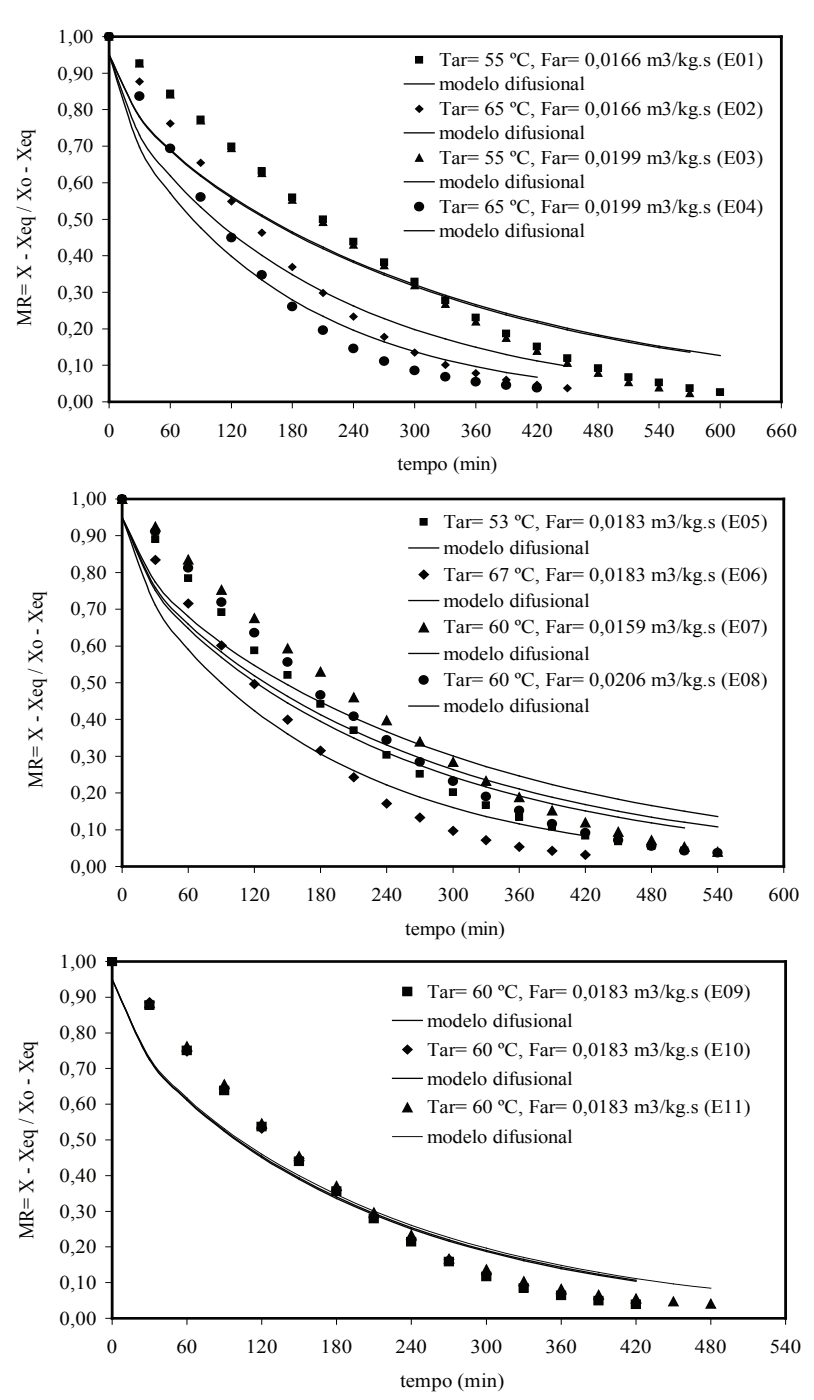

Figura 1. Curvas cinéticas de secagem da casca de mandioca com ar quente e modelagem do processo, segundo o modelo de Crank (A: ensaios E01, E02, E03 e E04; B: E05, E06, E07 e E08; C: E09, E10 e E11) (Goiânia, GO, 2011).

temente, o aumento da $D_{e f}$ Os valores de $D_{e f}$ estão plenamente em concordância com os dados de taxa de secagem, uma vez que a $D_{e f}$ representa a velocidade com a qual a água é retirada do interior do material.

Assim como neste trabalho, vários outros autores também utilizaram essa técnica para avaliar diferentes produtos alimentícios, tais como Ferreira \& Pena (2010), que estudaram a secagem da casca de maracujá amarelo proveniente de resíduos da industrialização de suco; Monteiro et al. (2010), que avaliaram a cinética de secagem do mesocarpo do maracujá amarelo, também proveniente de descarte da indústria; e Machado et al. (2010), que analisaram a cinética de secagem do pedúnculo de caju, a fim de

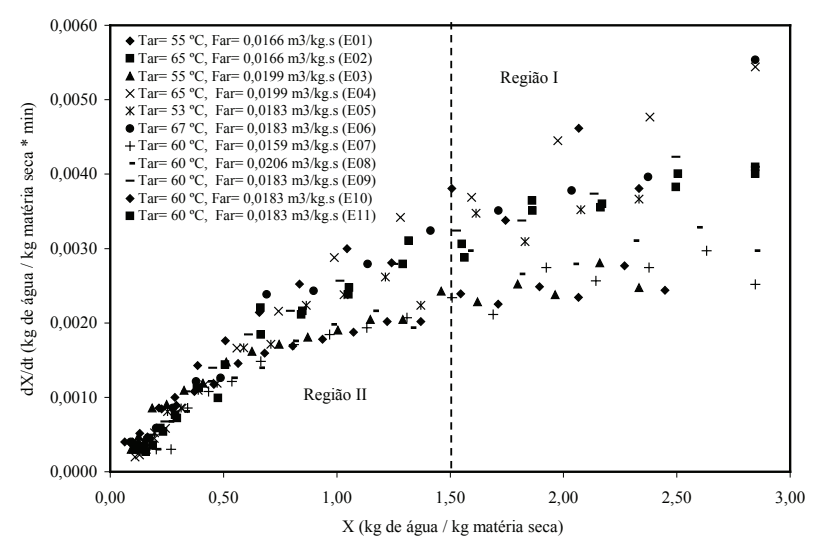

Figura 2. Taxa de secagem, em função da umidade em base seca, para as cascas de mandioca secas com ar quente (Goiânia, GO, 2011).

minimizar as perdas do fruto pelos produtores rurais. Martinazzo et al. (2007) realizaram a análise e descrição matemática da cinética de secagem de folhas de capim-limão, utilizando, também, o modelo para produtos de geometria plana, porém, não foi identificada, na literatura, referência à secagem de CM.

Os dados obtidos para o tempo de secagem $\left(\mathrm{T}_{\mathrm{s}}\right)$, parâmetros instrumentais de cor $\left(\mathrm{L}^{*}, \mathrm{a}^{*}\right.$ e a diferença de cor $(\Delta \mathrm{E})), \mathrm{pH}$ e acidez titulável das amostras de CM estão apresentados nas Tabelas 2 e 4 . Os níveis de significância (p) dos efeitos linear e quadrático e a interação da temperatura do ar $\left(\mathrm{T}_{\mathrm{ar}}\right)$ e fluxo do ar $\left(\mathrm{F}_{\mathrm{ar}}\right)$ sobre as variáveis dependentes estudadas, para o modelo completo e ajustado, além das regressões ajustadas com seus coeficientes de determinação, estão apresentados na Tabela 5, enquanto os gráficos das curvas de contorno dos modelos ajustados encontram-se ilustrados na Figura 3.

Com relação ao $\mathrm{T}_{\mathrm{s}}$ das $\mathrm{CM}$, o efeito quadrático da $\mathrm{T}_{\mathrm{ar}}$, linear do $\mathrm{F}_{\mathrm{ar}}$ e interação $\mathrm{T}_{\mathrm{ar}} \mathrm{F}_{\mathrm{ar}}$ não foram significativos $(\mathrm{p}>0,05)$ (Tabela 5). Com o aumento da $T_{a r}$, houve tendência de diminuição do $T_{s}$ (efeito linear $)$ da $\mathrm{CM}(\mathrm{p} \leq 0,05)$. $\mathrm{A} \mathrm{T}_{\mathrm{s}}$ da $\mathrm{CM}$ aumentou até valores de $\mathrm{F}_{\text {ar }}$ próximos a 0,0183 , depois diminuiu (efeito quadrático) $(\mathrm{p} \leq 0,05)$. O menor tempo de secagem (420 min.) da CM foi verificado quando $F_{\text {ar }}$ foi de $0,0183 \mathrm{~m}^{3} \mathrm{~kg}^{-1} \mathrm{~s}^{-1} \mathrm{e}$ a temperatura de $67^{\circ} \mathrm{C}$ (Figura 3).

Para a luminosidade da FCM, somente o efeito quadrático do $\mathrm{F}_{\text {ar }}$ foi significativo $(\mathrm{p} \leq 0,05)$, enquanto os demais efeitos não foram detectados $(\mathrm{p}>0,05)$. Entretanto, optou-se por manter o termo linear e o quadrático de $\mathrm{T}_{\mathrm{ar}}$, além da interação $\mathrm{T}_{\mathrm{ar}} \mathrm{F}_{\mathrm{ar}}$ no modelo 
Tabela 3. Difusividade efetiva $\left(D_{e f}\right)$, coeficiente de correlação $\left(R^{2}\right)$ e desvio relativo médio $(P)$ para o modelo difusional em casca de mandioca, em função da temperatura do ar $\left(\mathrm{T}_{\mathrm{ar}}\right)$ e fluxo do ar $\left(\mathrm{F}_{\mathrm{ar}}\right)$ (Goiânia, $\left.\mathrm{GO}, 2011\right)$.

\begin{tabular}{|c|c|c|c|c|c|c|}
\hline \multirow{2}{*}{ Ensaio } & $\mathrm{T}_{\mathrm{ar}}$ & $\mathrm{F}_{\mathrm{ar}}$ & $K$ & $D_{e f} \times 10^{9}$ & \multirow{2}{*}{$R^{2}$} & $P$ \\
\hline & ${ }^{\circ} \mathrm{C}$ & $\mathrm{m}^{3} \mathrm{~kg}^{-1} \mathrm{~s}^{-1}$ & $\left(\min .^{-1}\right)^{a}$ & $\mathrm{~m}^{2} \mathrm{~s}^{-1}$ & & $\%$ \\
\hline E01 & $55 \pm 0,5$ & 0,0166 & 0,00309 & 1,87 & 0,906 & 29,63 \\
\hline E02 & $65 \pm 0,5$ & 0,0166 & 0,00470 & 2,84 & 0,933 & 27,56 \\
\hline E03 & $55 \pm 0,5$ & 0,0199 & 0,00314 & 1,89 & 0,901 & 29,35 \\
\hline E04 & $65 \pm 0,5$ & 0,0199 & 0,00592 & 3,57 & 0,954 & 24,67 \\
\hline E05 & $53 \pm 0,5$ & 0,0183 & 0,00400 & 2,41 & 0,938 & 24,51 \\
\hline E06 & $67 \pm 0,5$ & 0,0183 & 0,00540 & 3,26 & 0,947 & 27,53 \\
\hline E07 & $60 \pm 0,5$ & 0,0159 & 0,00331 & 2,00 & 0,909 & 27,04 \\
\hline E08 & $60 \pm 0,5$ & 0,0206 & 0,00374 & 2,26 & 0,924 & 27,91 \\
\hline E09 & $60 \pm 0,5$ & 0,0183 & 0,00489 & 2,95 & 0,931 & 27,60 \\
\hline E10 & $60 \pm 0,5$ & 0,0183 & 0,00483 & 2,91 & 0,931 & 26,73 \\
\hline E11 & $60 \pm 0,5$ & 0,0183 & 0,00472 & 2,85 & 0,934 & 27,47 \\
\hline
\end{tabular}

${ }^{a}$ Constante de secagem.

Tabela 4. Parâmetros instrumentais de cor ( $\mathrm{L}^{*}, \mathrm{a}^{*}$ e $\left.\Delta \mathrm{E}\right), \mathrm{pH}$ e acidez titulável das amostras de farinha de casca de mandioca obtidas nos ensaios de 1 a 11 (Goiânia, GO, 2011).

\begin{tabular}{cccccc}
\hline Ensaio & $\mathrm{L}^{*}$ & $\mathrm{a}^{*}$ & $\Delta \mathrm{E}^{a}$ & $\begin{array}{c}\text { Acidez } \\
\text { titulável }\end{array}$ & $\mathrm{pH}$ \\
\hline E01 & 56,97 & 5,51 & 39,56 & 5,97 & 4,38 \\
E02 & 64,25 & 4,37 & 32,51 & 5,97 & 4,33 \\
E03 & 60,20 & 4,00 & 36,39 & 6,17 & 4,35 \\
E04 & 63,63 & 4,62 & 33,14 & 5,77 & 4,41 \\
E05 & 62,11 & 4,50 & 34,24 & 5,57 & 4,23 \\
E06 & 61,52 & 4,66 & 34,99 & 5,57 & 4,40 \\
E07 & 59,72 & 4,98 & 36,91 & 5,57 & 4,37 \\
E08 & 58,87 & 5,26 & 37,85 & 5,37 & 4,32 \\
E09 & 64,25 & 4,43 & 32,56 & 5,97 & 4,38 \\
E10 & 64,83 & 4,59 & 32,32 & 6,39 & 4,38 \\
E11 & 64,54 & 4,51 & 33,17 & 6,76 & 4,35 \\
\hline
\end{tabular}

${ }^{a}$ Diferença de cor.

ajustado (Tabela 5), o que aumentou o valor do coeficiente de determinação ajustado, possibilitando uma maior aproximação dos dados observados com os calculados pelo modelo.

Observou-se tendência ao clareamento do material, com o aumento de $\mathrm{T}_{\text {ar }}$ até $60^{\circ} \mathrm{C}$, e, em seguida, uma ligeira tendência de redução de $\mathrm{L}^{*}$. Com relação ao $\mathrm{F}_{\mathrm{ar}}$, notou-se forte clareamento da FCM até $0,0183 \mathrm{~m}^{3} \mathrm{~kg}^{-1} \mathrm{~s}^{-1}$, com $\mathrm{L}^{*}$ em queda, em valores de $F_{\text {ar }}$ superiores $(p \leq 0,05)$ (Figura $3 B$ ).

Pela análise de variância sobre a coordenada de cor a* da FCM, somente a interação $\mathrm{T}_{\mathrm{ar}} \mathrm{F}_{\mathrm{ar}}$ foi significativa $(\mathrm{p} \leq 0,05)$, mas o termo quadrático do $F_{a r}(p=0,055)$ foi mantido no modelo ajustado (Tabela 5). Ressalta-se que, na escala de cromati- cidade a* da FCM (Figura 3C), ocorreu pequena variação, ou que os níveis de $\mathrm{T}_{\mathrm{ar}}$ e $\mathrm{F}_{\mathrm{ar}}$ utilizados tiveram efeito reduzido sobre a mesma, chegando quase à neutralidade (zero), com leve tendência ao vermelho $(4,4-6,0)$.

A superfície obtida apresentou formato de sela, demonstrando que não houve um padrão específico de comportamento da cromaticidade $\mathrm{a}^{*}$, quando observados, isoladamente, a $\mathrm{T}_{\text {ar }}$ ou o $\mathrm{F}_{\text {ar }}$, mas que houve forte interação dos efeitos, quando combinados. Para o $F_{\text {ar }}$ de $0,0183 \mathrm{~m}^{3} \mathrm{~kg}^{-1} \mathrm{~s}^{-1}$, a cromaticidade a* foi, aproximadamente, constante, independentemente do valor de $\mathrm{T}_{\mathrm{ar}}$. Para valores de $\mathrm{F}_{\mathrm{ar}}$ menores que $0,0183 \mathrm{~m}^{3} \mathrm{~kg}^{-1} \mathrm{~s}^{-1}$, a cromaticidade a* da FCM decresceu com o aumento de $\mathrm{T}_{\mathrm{ar}}$, enquanto, para valores de $\mathrm{F}_{\text {ar }}$ maiores que $0,0183 \mathrm{~m}^{3} \mathrm{~kg}^{-1} \mathrm{~s}^{-1}$, aumentou com a elevação da $\mathrm{T}_{\mathrm{ar}}$ (Figura 3C). Tal comportamento pode ser decorrente da pequena variação da cromaticidade $\mathrm{a}^{*} \mathrm{e}$ da não indicação de um padrão de comportamento propriamente dito. Talvez seja mais prudente afirmar que a cromaticidade a* da FCM é, aproximadamente, constante, para a faixa de valores de $\mathrm{T}_{\mathrm{ar}}$ e $\mathrm{F}_{\mathrm{ar}}$ utilizados neste trabalho. Os fatores $\mathrm{T}_{\mathrm{ar}} \mathrm{e}$ $\mathrm{F}_{\mathrm{ar}}$ testados neste estudo não influenciaram na coordenada de cromaticidade $b^{*}$ da FCM.

$\mathrm{Na}$ análise de variância, somente o efeito quadrático de $\mathrm{F}_{\mathrm{ar}}$ durante a secagem, foi significativo $(\mathrm{p} \leq 0,05)$ para a diferença de cor $(\Delta \mathrm{E})$ da FCM, o mesmo ocorrendo para a acidez titulável (AT). Entretanto, por melhorar o coeficiente de determinação ajustado, foram mantidos, no modelo ajustado para $\Delta \mathrm{E}$, também os termos de $\mathrm{T}_{\mathrm{ar}}$ linear e quadrático e a interação $\mathrm{T}_{\mathrm{ar}} \mathrm{F}_{\mathrm{ar}}$ e $\mathrm{T}_{\mathrm{ar}}$ linear para AT (Tabela 5). 
Tabela 5. Níveis de significância dos efeitos linear e quadrático e interação da temperatura $\left(\mathrm{T}_{\mathrm{ar}}\right)$ e fluxo do ar $\left(\mathrm{F}_{\mathrm{ar}}\right)$ sobre o tempo de secagem $\left(\mathrm{T}_{\mathrm{s}}\right)$ da casca de mandioca, luninosidade $\left(\mathrm{L}^{*}\right)$, coordenadas de cromaticidade $\left(\mathrm{a}^{*}\right)$, diferença de cor $(\Delta \mathrm{E})$, acidez titulável e pH das farinhas de casca de mandioca, para o modelo completo e ajustado, além das regressões ajustadas, com seus coeficientes de determinação (Goiânia, GO, 2011).

\begin{tabular}{|c|c|c|c|c|c|c|c|}
\hline \multirow{2}{*}{$\begin{array}{l}\text { Variável } \\
\text { dependente }\end{array}$} & \multicolumn{5}{|c|}{$\begin{array}{l}\text { Nível de significância }(p) \text { obtido para cada efeito na } \\
\text { Anova do modelo completo/ajustado }\end{array}$} & \multirow{2}{*}{ Modelo ajustado } & \multirow{2}{*}{$\mathrm{R}^{2}$} \\
\hline & $\mathrm{T}_{\mathrm{ar}}(\mathrm{L})$ & $\mathrm{T}_{\mathrm{ar}}(\mathrm{Q})$ & $\mathrm{F}_{\mathrm{ar}}(\mathrm{L})$ & $\mathrm{F}_{\mathrm{ar}}(\mathrm{Q})$ & $\mathrm{T}_{\mathrm{ar}} \times \mathrm{F}_{\mathrm{ar}}$ & & \\
\hline $\mathrm{T}_{\mathrm{s}}$ & $\begin{array}{l}0,00896 / \\
0,00153\end{array}$ & $\begin{array}{c}0,39354 / \\
-\end{array}$ & $\begin{array}{c}0,58551 / \\
-\end{array}$ & $\begin{array}{l}0,01965 / \\
0,00614 \\
\end{array}$ & $\begin{array}{c}1,00000 / \\
-\end{array}$ & $y=453,47-53,53 x_{1}+47,91 x_{2}^{2}$ & 0,81710 \\
\hline $\mathrm{L}^{*}$ & $\begin{array}{c}0,13831 / \\
0,10349\end{array}$ & $\begin{array}{c}0,21590 / \\
0,17411\end{array}$ & $0,81149 /$ & $0,03279 /$ & $\begin{array}{c}0,37589 / \\
0,33093\end{array}$ & $y=64,54+1,24 x_{1}-1,28 x_{1}^{2}-2,45 x_{2}^{2}-0,96 x_{1} x_{2}$ & 0,71900 \\
\hline$a^{*}$ & $\begin{array}{c}0,75576 / \\
-\end{array}$ & $0,87551 /$ & $\begin{array}{c}0,38916 / \\
-\end{array}$ & $\begin{array}{l}0,12828 / \\
0,05445\end{array}$ & $0,04213 /$ & $\mathrm{y}=4,49+0,26 \mathrm{x}_{2}^{2}+0,44 \mathrm{x}_{1} \mathrm{x}_{2}$ & 0,64980 \\
\hline$\Delta \mathrm{E}$ & $\begin{array}{c}0,15495 / \\
0,11778\end{array}$ & $\begin{array}{c}0,36899 / \\
0,32330\end{array}$ & $\begin{array}{c}0,83456 / \\
-\end{array}$ & $\begin{array}{l}0,04430 / \\
0,02692\end{array}$ & $\begin{array}{c}0,37570 / \\
0,33010\end{array}$ & $y=32,69-1,16 x_{1}+0,82 x_{2}+2,27 x_{2}^{2}+0,95 x_{1} x_{2}$ & \\
\hline $\begin{array}{l}\text { Acidez } \\
\text { titulável }\end{array}$ & $\begin{array}{c}0,73027 / \\
-\end{array}$ & $\begin{array}{l}0,13830 / \\
0,06591\end{array}$ & $\begin{array}{c}0,80735 / \\
-\end{array}$ & $\begin{array}{l}0,09372 / \\
0,03696 \\
\end{array}$ & $\begin{array}{c}0,62853 / \\
-\end{array}$ & $y=6,37-0,29 x_{1}^{2}-0,34 x_{2}^{2}$ & 0,51200 \\
\hline $\mathrm{pH}$ & $\begin{array}{c}0,12984 / \\
0,06261 \\
\end{array}$ & $\begin{array}{c}0,42032 / \\
-\end{array}$ & $\begin{array}{c}0,88759 / \\
-\end{array}$ & $\begin{array}{c}0,88971 / \\
-\end{array}$ & $\begin{array}{c}0,31097 / \\
0,22247\end{array}$ & $\mathrm{y}=4,354+0,031 \mathrm{x}_{1}+0,028 \mathrm{x}_{1} \mathrm{x}_{2}$ & 0,43940 \\
\hline
\end{tabular}
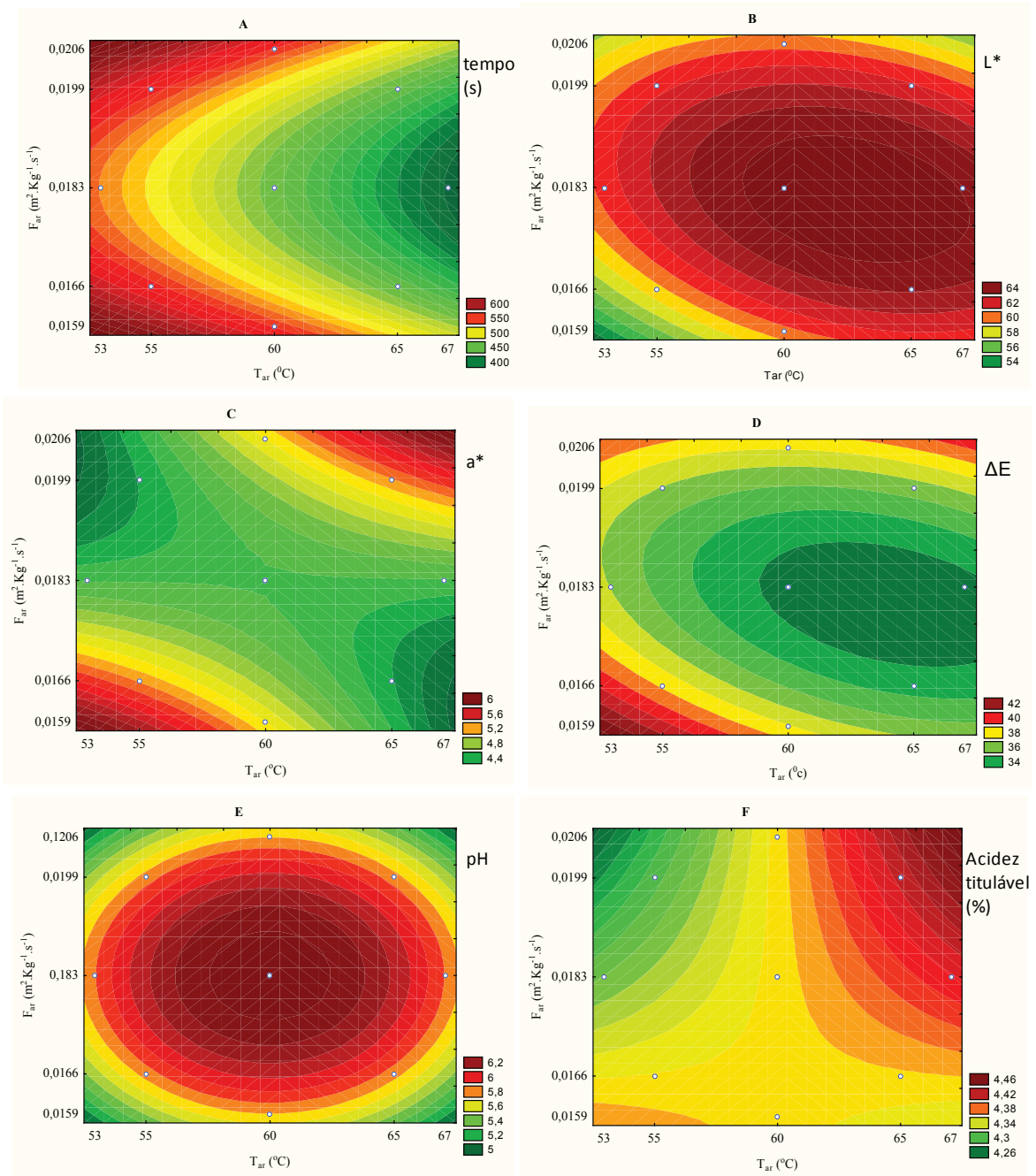

Figura 3. Curvas de contorno do tempo de secagem (A), luminosidade (L*)(B), cromaticidade (a*) (C), diferença de cor $\Delta \mathrm{E}(\mathrm{D})$, acidez titulável (E) e pH (F) (Goiânia, GO, 2011). 
Nas curvas de contorno da Figura 3D, observou-se que o efeito linear da $\mathrm{T}_{\mathrm{ar}}$ apresentou ligeira tendência em aumentar a $\Delta \mathrm{E}$, e que o efeito quadrático do $\mathrm{F}_{\mathrm{ar}}$ foi bem marcante na diferença de cor, entre o início e o final da secagem da FCM $(\mathrm{p} \leq 0,05)$, pois os valores de $\Delta \mathrm{E}$ diminuíram, com a elevação do $\mathrm{F}_{\mathrm{ar}}$ até $0,0183 \mathrm{~m}^{3} \mathrm{~kg}^{-1} \mathrm{~s}^{-1}$, para tornar a aumentar após este nível, enquanto, para a AT, observou-se região de máxima acidez próxima ao ponto central (6,2\%). Portanto, com a elevação do $\mathrm{F}_{\mathrm{ar}}$, durante a secagem, houve diminuição da AT da FCM até os valores intermediários estudados, com diminuição a partir daí $(\mathrm{p} \leq 0,05)$, o mesmo ocorrendo com a $\mathrm{T}_{\mathrm{ar}}$, embora o nível de significância tenha sido $6,6 \%$. Farinhas com menor AT, geralmente, apresentam qualidade superior, em relação aos aspectos sensoriais, também com produção de massa menos ácida.

A faixa de variação do $\mathrm{pH}$ foi pequena $(4,25 \mathrm{a}$ $4,45)$, devido ao fato de os níveis das variáveis testadas neste estudo não terem influenciado no $\mathrm{pH}$ da FCM, durante a secagem $(p>0,05)$. Mesmo assim, o resultado foi apresentado somente para verificação da tendência da resposta e devido à importância da mesma para a qualidade físico-química da FCM. Para valores de $\mathrm{F}_{\mathrm{ar}}$ maiores que $0,0166 \mathrm{~m}^{3} \mathrm{~kg}^{-1} \mathrm{~s}^{-1}$, houve ligeiro aumento do $\mathrm{pH}$ nas $\mathrm{T}_{\mathrm{ar}}$ acima de $65^{\circ} \mathrm{C}$, e comportamento inverso nas $\mathrm{T}_{\text {ar }}$ abaixo de $55^{\circ} \mathrm{C}$, ou seja, redução com a elevação do $\mathrm{F}_{\text {ar }}$ (Figura $3 \mathrm{~A}$ ).

\section{CONCLUSÕES}

1. O modelo de Crank para geometria plana é aplicável para modelar a cinética de secagem da casca de mandioca em secador convectivo de bandejas. Quanto maior a temperatura do ar, e nas condições intermediárias de fluxo de ar utilizadas, maior foi a taxa de secagem das CM.

2. A melhor condição de secagem das cascas de mandioca foi a combinação da temperatura do ar de $67^{\circ} \mathrm{C}$ e fluxo do ar de $0,0183 \mathrm{~m}^{3} \mathrm{~kg}^{-1} \mathrm{~s}^{-1}$, pois obteve-se o menor tempo de secagem e a melhor qualidade da farinha de casca de mandioca (desenvolvida a partir da CM desidratada), com cor mais clara e menos avermelhada, menor variação de cor final durante a secagem, menor acidez titulável e maior $\mathrm{pH}$.

\section{REFERÊNCIAS}

AFONSO JÚNIOR, P. C.; CORRÊA, P. C. Comparação de modelos matemáticos para descrição da cinética de secagem em camada fina de sementes de feijão. Revista Brasileira de Engenharia Agrícola e Ambiental, Campina Grande, v. 3, n. 3, p. 349-353, 1999.

AGUERRE, R. J.; SUAREZ, C.; VIOLLAZ, P. E. New BET type multilayer sorption isotherms: part II: modeling water sorption in foods. Lebensmittel Wissenschaft und Technologie, Zürich, v. 22, n. 4, p. 192-195, 1989.

ÁLVARES, V. S. et al. Análise da coloração da casca de banana 'Prata' tratada com etileno exógeno pelo método químico e instrumental. Revista Brasileira de Produtos Agroindustriais, Campina Grande, v. 5, n. 2, p. 155-160, 2003.

ANDRADE, E. T. et al. Cinética de secagem e qualidade de sementes de feijão. Engevista, Niterói, v. 8, n. 2, p. 8395, 2006.

BARROS NETO, B.; SCARMINO, I. S.; BRUNS, R. E. Planejamento e otimização de experimentos. Campinas: Ed. da Unicamp, 1996.

BRASIL. Resolução RDC n ${ }^{\circ}$ 263, de 22 de setembro de 2005. Regulamento técnico para produtos de cereais, amidos, farinhas e farelos. Diário Oficial da União, Brasília, DF, 23 set. 2005. Seção 1, p. 368-369.

CEREDA, M. P. Caracterização, usos e tratamentos de resíduos da industrialização da mandioca. Botucatu: Centro de Raízes Tropicais, 1996.

CEREDA, M. P. (Coord.). Culturas de tubersosas amiláceas latinoamericanas: propriedades gerais do amido. Campinas: Fundação Cargill, 2001.

CORRÊA, J. L. G. et al. Analysis of osmotic dehydration variables: influences on tomato (Licopersicon esculentum) drying. Boletim do Centro de Pesquisa e Processamento de Alimentos, Curitiba, v. 25, n. 2, p. 315-328, 2007.

CORRÊA, J. L. G. et al. Desidratação osmótica de tomate seguida de secagem. Revista Brasileira de Produtos Agroindustriais, Campina Grande, v. 10, n. 1, p. 35-42, 2008.

CRANK, J. The mathematics of diffusion. 2. ed. Oxford: Claredon Press, 1975.

DEMIREL, D.; TURHAN, M. Air-drying behavior of Dwarf Cavendish and Gross Michel banana slices. Journal of Food Engineering, London, v. 59, n. 1, p. 1-11, 2003.

DIAS, L. T.; LEONEL, M. Caracterização físico-química de farinhas de mandioca de diferentes localidades do Brasil. Ciência e Agrotecnologia, Lavras, v. 30, n. 4, p. 692-700, 2006.

DOYMAZ, I. Convective air drying characteristics of thin layer carrots. Journal of Food Engineering, London, v. 61, n. 1, p. 359-364, 2004. 
EL-SHARKAWY, M. A. International research on cassava photosynthesis, productivity, eco-physiology, and responses to environmental stresses in the tropics. Photosynthetica, Praha, v. 44, n. 4, p. 481-512, 2006.

FERREIRA, M. F. P.; PENA, R. S. Estudo da secagem da casca do maracujá amarelo. Revista Brasileira de Produtos Agroindustriais, Campina Grande, v. 12, n. 1, p. 15-28, 2010.

GIRALDO-ZUNIGA, A. D. et al. Drying kinetics for murici (Byrsonima crassifolia) fruit. Journal of Food Processing and Preservation, Malden, v. 30, n. 1, p. 699705, 2006.

INSTITUTO ADOLFO LUTZ. Normas analíticas do Instituto Adolfo Lutz: métodos físico-químicos para análise de alimentos. 4. ed. Brasília, DF: Ministério da Saúde/ Agência Nacional de Vigilância Sanitária, 2005.

KAYA, A.; AYDIN, O.; DINCER, I. Experimental and numerical investigation of heat and mass transfer during drying of Hayward kiwi fruits (Actinidia Deliciosa Planch). Journal of Food Engineering, London, v. 88, n. 3, p. 323-330, 2008.

KROKIDA, M. K. et al. Drying kinetics of some vegetables. Journal of Food Engineering, London, v. 59, n. 4, p. 391-403, 2003.

LACERDA, L. G. et al. Thermoanalytical and starch content evaluation of cassava bagasse as agro-industrial residue. Brazilian Archives of Biology and Technology, Curitiba, v. 52, n. 1, p. 143-150, 2009.

LAHSASNI, S. et al. Drying kinetics of prickly pear fruit (Opuntia ficus Indica). Journal of Food Engineering, London, v. 61, n. 1, p. 173-179, 2004.

LOMAURO, C. J.; BAKSHI, A. S.; LABUSA, T. P. Evaluation of food moisture sorption isotherm equations: part I: fruit, vegetable and meat products. Lebensmittel Wissenschaft und Technologie, Zürich, v. 18, n. 1, p. 112122, 1985.

MACHADO, A. V. et al. Estudo cinético da secagem do pedúnculo de caju em um secador convencional. Revista Verde de Agroecologia e Desenvolvimento Sustentável, Limoeiro, v. 6, n. 1, p. 36-42, 2010.

MARQUES, J. A. et al. Avaliação da mandioca e seus resíduos industriais em substituição ao milho no desempenho de novilhas confinadas. Revista Brasileira de Zootecnia, Viçosa, v. 29, n. 5, p. 1528-1536, 2000.

MARTINAZZO, A. P. et al. Análise e descrição matemática da cinética de secagem de folhas de capim-limão. Revista Brasileira de Engenharia Agrícola e Ambiental, Campina Grande, v. 11, n. 3, p. 301-306, 2007.

MONTEIRO, L. B. et al. Curva de secagem do mesocarpo do maracujá amarelo. Revista Ceciliana, Santos, v. 2, n. 1, p. 42-44, 2010.
NAGLE, M. et al. Effects of operating practices on performance of a fixed-bed convection dryer and quality of dried longan. Journal of Food Science and Technology, New York, v. 43, n. 1, p. 1979-1987, 2008.

PARK, K. J.; BIN, A.; BROD, F. P. R. Drying of pear d'Anjou with and without osmotic dehydration. Journal of Food Engineering, London, v. 56, n. 1, p. 97-103, 2002.

PRADO, I. N. et al. Desempenho de novilhas alimentadas com rações contendo milho ou casca de mandioca como fonte energética e farelo de algodão ou levedura como fonte proteica. Revista Brasileira de Zootecnia, Viçosa, v. 29 , n. 1, p. 278-287, 2000.

QUEIROZ, M. R.; NEBRA, S. A. Theoretical and experimental analysis of drying kinetics of bananas. Journal of Food Engineering, London, v. 47, n. 2, p. 127 132, 2001.

RESENDE, O.; FERREIRA, L. U.; ALMEIDA, D. P. Modelagem matemática para descrição da cinética de secagem do feijão Adzuki (Vigna angularis). Revista Brasileira de Produtos Agroindustriais, Campina Grande, v. 12, n. 2, p. 171-178, 2010.

SIMAL, S.; DEYÁ, E.; ROSELLÓ, C. Simple modeling of drying curves of fresh and osmotically pre-dehydrated apple cubes. Journal of Food Engineering, London, v. 33, n. 1, p. 139-150, 1997.

SIMAL, S. et al. Use of exponential, Page's and diffusional models to simulate the drying kinetics of kiwi fruit. Journal of Food Engineering, London, v. 43, n. 1, p. 109-114, 2005.

SOUZA, L. S.; FIALHO, J. F. Cultivo da mandioca para a região do Cerrado. 2003. Disponível em: $<\mathrm{http}: / /$ sistemasdeproducao.cnptia.embrapa.br/>. Acesso em: maio 2012.

SOUZA, T. A. C. Segurança microbiológica dos resíduos de fecularia e aplicação em bolos para a alimentação humana. 2011. 78 f. Dissertação (Mestrado em Ciência e Tecnologia de Alimentos) - Escola de Agronomia e Engenharia de Alimentos, Universidade Federal de Goiás, Goiânia, 2011.

STASOFT. Statistica 7.0 for Windows: computer program manual. Tulsa: Statsoft Inc., 2007. 1 CD-ROM.

VILHALVA, D. A. A. et al. Aproveitamento da farinha de casca de mandioca na elaboração de pão de forma. Revista do Instituto Adolfo Lutz, São Paulo, v. 70, n. 4, p. 514-521, 2011.

VILPOUX, O.; CEREDA, M. P. Caracterização das fecularias no Brasil. Botucatu: Centro de Raízes Tropicais/ Unesp, 1995. 